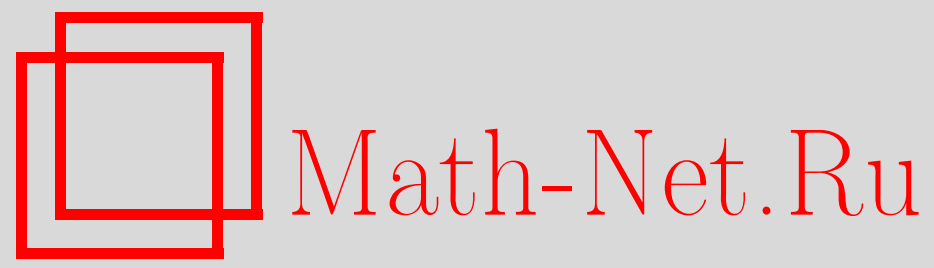

О. В. Грошев, Задача Коши для волнового уравнения на неглобально гиперболических многообразиях, Вестн. Сам. гос. техн. ун-та. Сер. Физ.-мат. науки, 2011, выпуск 1(), 42-46

DOI: https://doi.org/10.14498/vsgtu898

Использование Общероссийского математического портала Math-Net.Ru подразумевает, что вы прочитали и согласны с пользовательским соглашением http://www.mathnet.ru/rus/agreement

Параметры загрузки:

IP: 18.234 .197 .8

26 апреля 2023 г., $14: 18: 35$ 
УДК 517.95

\section{ЗАДАЧА КОШИ ДЛЯ ВОЛНОВОГО УРАВНЕНИЯ НА НЕГЛОБАЛЬНО ГИПЕРБОЛИЧЕСКИХ МНОГООБРАЗИЯХ}

\section{О. В. Грошев}

Математический институт им. В. А. Стеклова РАН, 119991, Москва, ул. Губкина, 8.

E-mail: groshev@mi.ras.ru

Рассматривается задача Коши для волнового уравнения на двух типах неглобально гиперболических многообразий: плоскости Минковского с присоединенной ручкой и пространстве Мизнера. Доказано, что на плоскости с ручкой существование и единственность классического решения равносильны конечному набору точечных условий на начальные данные. На пространстве Мизнера существование и единственность классического решения эквивалентны гораздо более ограничивающим условиям на начальные данные.

Ключевые слова: волновое уравнение, задача Коши, неглобально гиперболические многообразия.

Введение. Теория (не)глобально гиперболических многообразий имеет два источника. Во-первых, это работы Петровского и Лерэ по теории гиперболических уравнений $[1,2]$, в которых было доказано, что задача Коши для волнового уравнения

$$
\square u=\frac{1}{\sqrt{-g}} \partial_{\alpha}\left(g^{\alpha \beta} \sqrt{-g} \partial_{\beta} u\right)=0
$$

всегда разрешима для некоторого специального типа многообразий, названных глобально гиперболическими. Во-вторых, в Общей теории относительности возникли многообразия с нарушением причинной структуры, которые не являются глобально гиперболическими. Таковы, например, пространство анти-де Ситтера, Гёделя и Готта, машина времени Дойча-Полицера и многие другие [3-5].

Глобально гиперболическим многообразием называется ориентируемое по времени лоренцево многообразие $(M, g)$, которое не имеет замкнутых времениподобных кривых, и множество времениподобных путей между любыми двумя точками которого компактно. С физической точки зрения наличие замкнутых времениподобных кривых означает нарушение причинности и соответствует возможности перемещения во времени. Берналь и Санчес доказали [6], что все глобально гиперболические многообразия диффеоморфны $\mathbb{R}^{1} \times \Sigma$, где $\Sigma$ - поверхность Коши.

Задача Коши для волнового уравнения на неглобально гиперболических многообразиях рассматривалась в [7-10]. В частности, в последней работе доказаны существование и единственность классического решения уравнения Клейна-Гордона на факторе пространства анти-де Ситтера, а также на кротовых норах специального класса.

Настоящая работа является продолжением работы [11], выполненной вместе с соавторами, а так же [12]. Было доказано, что на плоскости Минковско-

Олег Викторинович Грошев, аспирант, отд. математической физики. 
го с присоединенной ручкой (модифицированная машина Дойча-Полицера) классическое решение существует и единственно при выполнении конечного набора точечных условий самосогласованности; кроме того, в работе [11] были приведены примеры, когда полученное решение путешествует во времени, а также затронут вопрос существования обобщённых решений. Результаты для пространства Мизнера существенно отличаются от результатов для плоскости с ручкой. Доказано, что существование классического решения на нем эквивалентно таким условиям на начальные данные, которые в точности запрещают путешествия во времени. Данная работа мотивирована изучением возможности создания кротовых нор и неглобально гиперболических регионов при столкновении частиц на высоких энергиях [13].

1. Результаты для плоскости с ручкой. Приведём основные результаты, полученные для плоскости с ручкой. Для начала опишем конструкцию плоскости с ручкой.

На полуплоскости $\mathbb{R}_{+}^{2}=\left\{(x, t) \in \mathbb{R}^{2} \mid t>0\right\}$ рассмотрим два вертикальных интервала $S_{1}$ и $S_{2}$ длины $\ell>0$ :

$$
S_{i}=\left\{(x, t) \in \mathbb{R}_{+}^{2} \mid x=x_{i}, \quad t_{i}<t<t_{i}+\ell\right\} .
$$

Предположим, что

$$
0<x_{2}-x_{1}<t_{2}-t_{1}+\ell
$$

таким образом, вектор $I=\left(x_{2}-x_{1}, t_{2}-t_{1}\right)$, переводящий $S_{1}$ в $S_{2}$, времениподобен.

Склеим стороны отрезков, как показано на рис. 1 , а именно склеим «внутренние» стороны разрезов друг с другом; аналогично склеим «внешние» стороны разрезов друг с другом. Получившееся многообразие имеет две особые точки - концы отрезков.

Любое гладкое поле, заданное на рассматриваемом многообразии, будет удовлетворять определённым условиям склейки на разрезах $S_{1}$ и $S_{2}$. Обратно, если поле дифференцируемо в области $\Omega=\mathbb{R}_{+}^{2} \backslash \bar{S}_{1} \cup \bar{S}_{2}$ и удовлетворяет

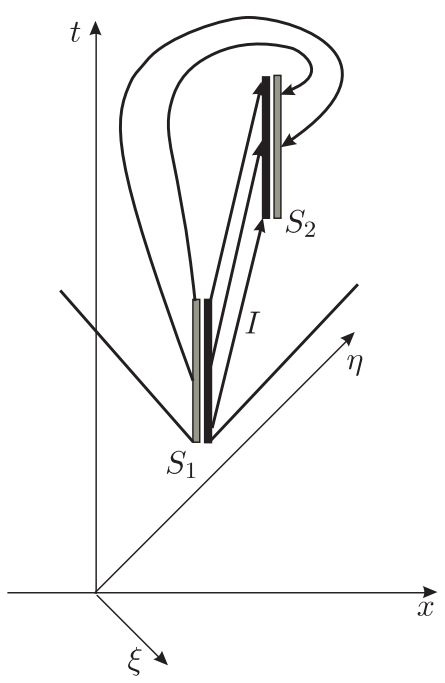

Рис. 1. Плоскость Минковского с двумя разрезами, склеенными определённым образом. Идентификация точек «внешних» и «внутренних» сторон разрезов указана линиями со стрелками этим условиям склейки, то оно гладко на рассматриваемом многообразии.

Рассмотрим волновое уравнение на этом многообразии

$$
u_{t t}-u_{x x}=0 \quad \text { в } \Omega
$$

с начальными условиями

$$
\left.u\right|_{t=0}=\varphi,\left.\quad u_{x}\right|_{t=0}=\psi,
$$

где $\varphi \in C^{2}(\mathbb{R}), \psi \in C^{1}(\mathbb{R})$. Наложим следующие условия склейки:

$$
u\left(X_{ \pm}\right)=u\left(X_{\mp}+I\right) \quad u_{x}\left(X_{ \pm}\right)=u_{x}\left(X_{\mp}+I\right),
$$


где $X=(x, t) \in S_{1}$, а $u\left(X_{ \pm}\right)=\lim _{x \rightarrow x_{1} \pm 0} u(x, t)$; предполагается, что указанные пределы справа и слева существуют.

ОПредЕЛЕниЕ 1.1. Классическим решением задачи (3)-(5) называется функция $u \in C^{2}(\Omega) \cap C^{1}(\Omega \cup\{t=0\})$, удовлетворяющая условиям (3)-(5) в предположении, что указанные в (5) пределы справа и слева существуют.

Обозначим $c_{i}=x_{i}-t_{i}, d_{i}=x_{i}+t_{i}$, где $i$ может быть 1 или 2. Будем обозначать через $\Phi$ следующий (упорядоченный) набор функций:

$$
\Phi(x)=\left(\Phi^{(i)}(x), i=0,1,2 ; \quad \int_{a}^{x} \psi(s) d s ; \quad \psi^{(j)}(x), j=0,1\right) .
$$

Обозначим через $\Psi$ следующий вектор:

$$
\Psi=\left(\Phi\left(c_{1}-\ell\right), \Phi\left(c_{2}-\ell\right), \Phi\left(c_{1}\right), \Phi\left(c_{2}\right), \Phi\left(d_{1}+\ell\right), \Phi\left(d_{2}+\ell\right), \Phi\left(d_{1}\right), \Phi\left(d_{2}\right)\right) .
$$

Теорема 1.1. Для существования классического решения задачи (3)-(5) необходимо и достаточно выполнение десяти линейно независимых условий, которые кратко можно записать как $L \Psi=0$, где $L$-некоторая специальная матрица ранга 10.

2. Результаты для пространства Мизнера. Пространство Мизнера - плоское двумерное пространство-время, являющееся фактором $\mathbb{R}^{1,1} /\langle\mathcal{B}\rangle$ пространства Минковского по свободной группе, порожденной бустом $\mathcal{B}$. В системе координат $(\xi, \eta)$, где $\xi=-(x+t), \eta=x-t$, буст $\mathcal{B}$ даётся следующим образом:

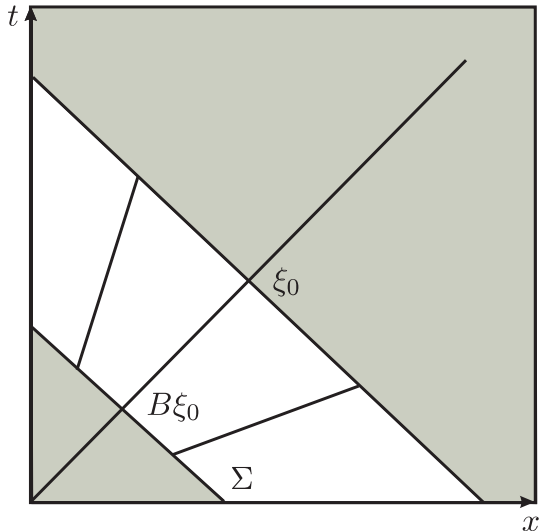

Рис. 2. Пространство Мизнера получается после отождествления границ фундаментальной области (на рисунке белая). После отождествления отрезки, отмеченные на фундаментальной области, превращаются в окружности

$$
\mathcal{B}:(\xi, \eta) \mapsto\left(B \xi, B^{-1} \eta\right), B \in \mathbb{R}_{+} .
$$

Он сохраняет Лоренцеву метрику $d s^{2}=$ $=-d \xi d \eta$. В качестве фундаментальной области действия $\mathcal{B}$ можно взять полосу $\left\{\xi_{0}<\xi<B \xi_{0}\right\}$. Само пространство Мизнера (см. рис. 2) получится, если у фундаментальной области отождествить границы с помощью

$$
\left(\xi_{0}, \eta\right) \sim\left(B \xi_{0}, B^{-1} \eta\right)
$$

На получившемся многообразии существует единственная замкнутая нулевая геодезическая $\eta=0$. Она делит пространство на два региона: «прошлое» и «будущее». В прошлом существуют глобальные поверхности Коши и не существует замкнутых времени подобных кривых; тогда как в будущем через каждую точку проходит замкнутая времениподобная кривая.

Волновое уравнение $\square u=0$ в координатах $(\xi, \eta)$ имеет вид:

$$
u_{\xi \eta}=0 \quad \text { в } \Theta=\left\{\xi_{0}<\xi<B \xi_{0}, t>0\right\} .
$$


Начальные данные задаются на поверхности Коши, которая лежит в прошлом, и поэтому является пространственно-подобной. В качестве такой поверхности можно взять $\Sigma=\{t=0\}$. Итак, задача Коши состоит в задании $u$ и $\nabla u$ на $\Sigma$ :

$$
\left.u\right|_{t=0}=\Phi,\left.\quad \nabla u\right|_{t=0}=\psi
$$

Кроме того, необходимо задать условия склейки $u$ на границах фундаментальной области. Они задаются следующим образом:

$$
u\left(\xi_{0}, \eta\right)=u\left(B \xi_{0}, B^{-1} \eta\right), \quad \nabla u\left(\xi_{0}, \eta\right)=\mathcal{B} \nabla u\left(B \xi_{0}, B^{-1} \eta\right) .
$$

ОПРЕДЕЛЕнИЕ 2.1. Классическим решением задачи (6)-(8) называется функция $u \in C^{2}(\Theta) \cap C^{1}(\bar{\Theta})$, удовлетворяющая условиям (6)-(8).

Теорема 2.1. Для существования классического решения задачи (6)-(8) необходимо и достаточно выполнение следующих условий:

$$
\Phi\left(\xi_{0}\right)=\Phi\left(B \xi_{0}\right), \quad \psi\left(\xi_{0}\right)=B \psi\left(B \xi_{0}\right), \quad \Phi^{\prime}=\psi .
$$

При этом классическое решение единственно и имеет вид волны, идущей влево $и(\xi, \eta)=\Phi(\xi)$.

ЗАмечАние 2.1.Качественно решение такого вида сильно отличаются от решений из п. 1. А именно, такие решения не путешествуют во времени, поскольку имеют только левую моду, которая не идет вдоль замкнутых времениподобных кривых.

Автор выражает благодарность И. В. Воловичу и участникам семинара НОЦ МИАН за полезные обсуждения.

Работа выполнена при поддержке программы «Ведущие научные школы» (проекты НШ-7675.2010.1, НШ-8784.2010.1) и РФФИ (проект 09-01-12161-офи-м).

\section{БИБЛИОГРАФИЧЕСКИЙ СПИСОК}

1. Petrowsky I. G. Über das Cauchysche Problem für Systeme von partiellen Differentialgleichungen // Mat. Sb., 1937. Vol.2(44), no. 5. Pp. 815-870.

2. Лере Ж. Гиперболические дифференциальные уравнения. М.: Наука, 1984. 208 с. [Lere Zh. Hyperbolic differential equations. Moscow: Nauka, 1984. 208 pp.]

3. Hawking S. W., Ellis G.F.R. The large scale structure of space-time/ Cambridge Monographs on Mathematical Physics. Vol. 1. London - New York: Cambridge University Press, 1973. 391 рр.; русск. пер.: Хокинг С., Эллис Дж. Крупномасштабная структура пространства-времени. М.: Мир, 1977. 432 с.

4. Politzer H. D. Path integrals, density matrices, and information flow with closed timelike curves // Phys. Rev. D, 1994. Vol. 49, no. 8. Pp. 3981-3989, arXiv: gr-qc/9310027.

5. Gott J.R. Closed timelike curves produced by pairs of moving cosmic strings: Exact solutions // Phys. Rev. Lett., 1991. Vol.66, no. 9. Pp. 1126-1129.

6. Bernal A., Sanchez A. Smoothness of time functions and the metric splitting of globally hyperbolic space-times // Comm. Math. Phys., 2005. Vol. 257, no. 1. Pp. 43-50.

7. Friedman J., Morris M.S., Novikov I.D., Echeverria F., Klinkhammer G., Thorne K.S., Yurtsever $U$. Cauchy problem in spacetimes with closed timelike curves // Phys. Rev. D, 1990. Vol. 42, no. 6. Pp. 1915-1930. 
8. Арефъева И. Я., Волович И. В., Ишиватари Т. Задача Коши на неглобально гиперболических многообразиях // ТMФ, 2008. Т. 157, № 3. С. 334-344; англ. пер.: Aref'eva I. Ya., Ishiwatari T., Volovich I. V. Cauchy problem on non-globally hyperbolic space-times // Theoret. and Math. Phys., 2008. Vol. 157, no. 3. Pp. 1646-1654, arXiv: 0903.0567 [hep-th].

9. Friedman J. L. The Cauchy problem on spacetimes that are not globally hyperbolic/ In: The Einstein equations and the large scale behavior of gravitational fields, 50 Years of the Cauchy problem in general relativity; P. T. Chrusćiel et al. New York: Birkhäuser, 2004. Pp. 331-346, arXiv: gr-qc/0401004.

10. Friedman J.L., Morris M.S. Existence and uniqueness theorems for massless fields on a class of spacetimes with closed timelike curves // Comm. Math. Phys., 1997. Vol. 186, no. 3. Pp. 495-530, arXiv: gr-qc/9411033.

11. Волович И. В., Грошев О.В., Гусев Н.А., Куръянович Э.А. О решениях волнового уравнения на неглобально гиперболическом многообразии / В сб.: Избранные вопросы математической физики и р-адического анализа: Сборник статей / Тр. МИАН, Т. 265. М.: МАИК, 2009. С. 273-287; англ. пер.: Volovich I. V., Groshev O. V., Gusev N. A., Kuryanovich E.A. On solutions to the wave equation on a non-globally hyperbolic manifold // Proc. Steklov Inst. Math., 2009. Vol. 265. Pp. 262-275, arXiv: 0903.0741 [hep-th].

12. Грошев О.В. О существовании и единственности классических решений задачи Коши на неглобально гиперболических многообразиях // TMФ, 2010. Т. 164, № 3. С. 441-446; англ. пер.: Groshev O.V. Existence and uniqueness of classical solutions of the Cauchy problem on nonglobally hyperbolic manifolds // Theoret. and Math. Phys., 2010. Vol.164, no. 3. Pp. 1202-1207.

13. Aref'eva I. Ya., Volovich I. V. Time Machine at the LHC,// Int. J. Geom. Meth. Mod. Phys., 2008. Vol. 5, no. 4. Pp. 641-651, arXiv: 0710.2696 [hep-th].

Поступила в редакцию $21 / \mathrm{XII} / 2010$;

в окончательном варианте - 17/II/2011.

MSC: $35 \mathrm{~L} 05$

\section{CAUCHY PROBLEM FOR THE WAVE EQUATION ON NON-GLOBAL HYPERBOLIC MANIFOLDS}

\section{O. V. Groshev}

Steklov Mathematical Institute, Russian Academy of Sciences, 8, Gubkina st., Moscow, 119991, Russia.

E-mail: groshev@mi.ras.ru

We consider Cauchy problem for wave equation on two types of non-global hyperbolic manifolds: Minkowski plane with an attached handle and Misner space. We prove that the classical solution on a plane with a handle exists and is unique if and only if a finite set of point-wise constraints on initial values is satisfied. On the Misner space the existence and uniqueness of a solution is equivalent to much stricter constraints for the initial data.

Key words: wave equation, Cauchy problem, non-globally hyperbolic manifolds.

Original article submitted 21/XII/2010; revision submitted 17/II/2011.

Oleg V. Groshev, Postgraduate Student, Dept. of Mathematical Physics. 